Research Article

\title{
Feasibility of an 8-week Yoga Intervention among Middle-aged and Older Adults
}

Neha P. Gothe *, Emily Erlenbach

Kinesiology and Community Health University of Illinois at Urbana Champaign, Urbana, IL, USA; EMail: npg@illinois.edu

* Correspondence: Neha P. Gothe; E-Mail: npg@illinois.edu

Academic Editor: Marieke Van Puymbroeck

Special Issue: Yoga in Older Adults

OBM Geriatrics

2020 , volume 4 , issue 4

doi:10.21926/obm.geriatr.2004137
Received: August 13, 2020

Accepted: October 28, 2020

Published: November 06, 2020

\begin{abstract}
Feasibility studies play a critical role in determining whether the target population is likely to engage with larger scale efficacy and effectiveness trials. Feasibility data for yoga interventions designed and conducted with older adults is limited. In this paper we present feasibility and acceptability data from an 8-week randomized controlled trial (RCT) conducted with middle aged and older adults. Participants $n=118$ (Mean age: 62.0 years) were randomized to either a Hatha yoga or Stretching Control group with hour long group exercise classes held $3 x /$ week for 8 weeks. Herein we report feasibility and acceptability, including enrollment rates, attendance, attrition and adverse events, participant feedback and satisfaction data. Of the 265 adults screened, 118 were eligible and randomized. Session attendance was high for both groups (80.82 to $81.29 \%$ ) and only 10 people were lost to follow-up. Program satisfaction for both groups was high (4.8 and 4.86 out of 5) and no adverse events were reported. Participants' feedback regarding most and least helpful aspects of the program as well as suggestions for future yoga interventions are summarized. Overall, the yoga intervention was highly feasible and acceptable. The feasibility parameters from this trial can aid researchers in estimating desired sample sizes to successfully recruit, randomize and retain older adults in short- and long-term yoga based RCTs.
\end{abstract}

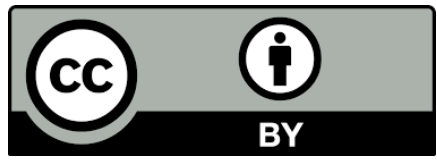

(C) 2020 by the author. This is an open access article distributed under the conditions of the Creative Commons by Attribution License, which permits unrestricted use, distribution, and reproduction in any medium or format, provided the original work is correctly cited. 


\section{Keywords}

Yoga intervention; RCT; feasibility; adherence

\section{Introduction}

Yoga is a holistic, mind-body activity that has components centering on meditation, breathing, and postures. Although its historical origins are in ancient Indian philosophy, it has gained popularity in Western countries. The recent National Health Interview Survey identified yoga as the most popular form of mind-body practice, used by $14.3 \%$ (32.5 million) U.S. adults [1]. The use of yoga and other complementary and alternative medicine therapies is becoming increasingly popular, especially among older adult populations who use these alternative therapies for aging-related chronic conditions such as back pain, arthritis, anxiety, depression, and cancer [2-5]. Although yoga practice involves physical postures that mimic stretching, balance, and strength exercises, resulting in physical benefits such as improved mobility and balance $[6,7]$, it also includes an active attentional component of breathing and meditation practice.

Although yoga continues to gain popularity among practitioners and researchers, preliminary studies that test its feasibility and acceptability, especially among older non-clinical adult populations, are limited. The few preliminary yoga studies which utilized healthy older adult samples have primarily studied fall-reduction, balance, and/or mobility outcomes $[6,8,9]$. Only two previous studies $[10,11]$ examined overall acceptance and tolerance of yoga practice among healthy older adults. However, Chen et al. [10] examined the acceptability and feasibility specific to newly developed yoga program (the Silver Yoga Programme), whereas Wang [11] focused on the ability to improve physical and mental well-being and did not focus on program feasibility metrics.

The National Center for Complementary and Integrative Health [12] suggests that feasibility studies are critical in informing the intervention design and can help determine whether participants can and will adhere to the yoga and assessment protocols such as attend all assessment visits, perform or tolerate the yoga intervention, identify and monitor adverse events, etc. Recruitment and retention methods are tested to demonstrate that the enrollment criteria identify the appropriate patient population and exclude those that cannot safely or appropriately participate in the yoga intervention or study related assessments. This is particularly important for physical activity interventions such as yoga for older adult populations, as safety of yoga practice and instruction is critical to designing yoga programs that are safe and modifiable for older adults with chronic health conditions, physical limitations or co-morbidities [13].

In this manuscript, we report the feasibility and acceptability data from an 8-week randomized controlled trial examining the effects of yoga vs. stretching and strengthening exercises on cognitive function and functional fitness among middle aged and older adults. The primary outcomes of cognitive function and functional fitness have been previously published [7, 14, 15]. Aligned with Eldridge and colleagues' [16] definition of a feasibility study, we aimed to include both "'developmental type questions" collected at the end of this trial in addition to quantitativelymeasured acceptability measures which may provide indications of success for future larger-scale trials. We evaluate the feasibility and acceptability parameters in the context of our 8-week yoga intervention, including: i) advertising and recruitment data such as recruitment rate, eligibility and 
enrollment rates; ii) yoga and active stretching-strengthening control group intervention feasibility data such as intensity, enjoyment, performance, attendance and adverse events, and iii) trial attrition rates and participant program feedback.

\section{Materials and Methods}

\subsection{Procedures and Intervention}

The primary study aim was to examine the efficacy of an 8-week yoga program to improve measures of cognition among older adults compared to a stretching-strengthening group which served as an active control. The primary and secondary outcomes and results have been published $[7,14,15]$, herein we report the feasibility and acceptability data from this trial. Briefly, eligibility criteria required participants to 1) be between 55-79 years of age, 2) speak English fluently, 3) engage in $<2$ days of structured physical activity for the last 6 months, 4) not currently practice yoga, and 5) be able to get up and down from the floor. Both the yoga and stretching-strengthening group met for three one-hour exercise sessions each week over eight weeks. Both intervention arms were designed to start at a beginner level and progress in intensity over the study period. The yoga group practiced Hatha yoga, and sessions were comprised of posture exercises (e.g. warrior pose, sun salutations, lotus pose), deep breathing exercises and meditation. The stretching-strengthening group was designed as an active control which focused on stretching and strengthening/toning for all major muscle groups aligned with the Physical Activity Guidelines for Americans.

\subsection{Measures}

\subsubsection{Advertising and Recruitment}

Participants were recruited between March 2012 and January 2013. The study was approved by the University of Illinois at Urbana Champaign's Institutional Review Board (IRB\# 12362). All participants signed a written informed consent before completing any study assessments. Recruitment was planned around a random-sampling approach. Methods of recruitment were limited to free advertising avenues and included emails through the University of Illinois LISTSERV, fliers, booths at community events, social media postings, and contacting participants in the lab database of volunteers. The efficacy of recruitment efforts to attract participants was analyzed via recruitment and enrollment rates. Recruitment rate was defined as the number of individuals who contacted the lab expressing interest in the study compared to the estimated total number of eligible individuals exposed to recruitment efforts. This estimate was calculated using information from the 2012 American Community Survey [17]. Enrollment rate was the number of individuals randomized into the program compared to the total number who initially contacted the lab. Additionally, we report 1) the percentage of individuals who passed pre-screening (i.e. met all the primary eligible criteria), and 2) the percentage of individuals who may have been eligible but could not be contacted or encountered logistical challenges preventing their enrollment. Total enrollment rate was calculated as the number study participants who completed follow-up out of those initially expressing interest in study participation. 


\subsubsection{Intervention Data}

At the end of each session over the 8-week study period, participants were asked to rank their enjoyment ("how much did you enjoy your activity session"), perceived intensity ("how hard did you feel like you were working"), and perceived performance ("how well do you think you performed today?"). Questions were asked using a either a 1-5 (for intensity and enjoyment) or 1-6 (for performance) Likert scale, with the highest value reflecting "very much" for enjoyment, "very hard" for perceived intensity, and "very well" for perceived performance. Given that the yoga sessions were divided into three components (poses, breathing, and meditation), participants in this group were asked to rank enjoyment, intensity, and perceived performance separately for each component of their practice. For the stretching group, participants were only asked to provide one rating of each measure for their session. Weekly averages for each of these measures were computed to capture participants' overall tolerance of their respective exercise group.

\subsubsection{Attendance, Attrition Rates and Safety}

Attendance rates for each group were calculated (excluding study dropouts) and an independent-samples t-test was conducted to examine group differences. The attrition rate across the entire study sample was calculated as the number of individuals who dropped-out of the study divided by the total number of randomized participants. Additionally, group-specific attrition rates were calculated and reasons for dropping out were summarized. Any adverse events which occurred during the study were also documented.

\subsubsection{Participant Program Feedback}

At the end of the program, participants were asked to provide a program evaluation. These questions related to overall experience and satisfaction with the class design and structure (i.e. exercises, progression, modifications). These questions were asked using a 1-5 Likert scale, with 5 being the highest rated ("very satisfied"). Additionally, participants were asked open-ended questions regarding the most and least beneficial aspects of the program and any suggestions they may have for future yoga or stretching programs.

\section{Results}

\subsection{Study Recruitment}

During the recruitment period, 265 people contacted the lab either via email or phone. Among those who enrolled in the study, emails via the University of Illinois LISTSERV was cited as the most common medium of advertising, followed by being part of a volunteer database in the lab, word of mouth and seeing study fliers in the community. We would like to note that no paid advertising was conducted for this trial and we estimate our advertising efforts to have reached approximately 20,000 residents (reach of the University listserv, in addition to the number of volunteers in the lab database) with as estimated recruitment rate of $1.325 \%(265 / 20,000)$. A total of $19.24 \%$ of the contacts (51/265) did not meet the primary eligibility criteria and were excluded. Being "too physically active" was the most common reason for exclusion during pre-screening. An additional $36.22 \%$ of the individuals $(96 / 265)$ were excluded for being "unable to contact" or for logistical 
reasons such as "time commitment" or time/day not being suitable for their study participation. Overall, $n=118$ individuals were successfully contacted, screened and randomized into one of the two study groups. This resulted in a randomization rate of $44.53 \%$ (118/265). Of the 118 participants, $\mathrm{n}=61$ and $\mathrm{n}=57$ were randomly assigned to either the yoga or stretching-strengthening arm, respectively. At the end of -weeks, 108 participants completed the study and all follow-up measures, producing a total enrollment rate of $40.75 \%$ (108/265).

\subsection{Baseline Characteristics of Study Participants}

As seen in Table 1, the average age of yoga participants was $62.1( \pm 5.82)$ and average age of stretching-strengthening participants was 62.0 ( \pm 5.39$)$. Out of the 118 participants initially randomized, $n=92$ (77.97\%) were female and $n=97$ (82.20\%) were Caucasian. We were successful in recruiting minority populations proportional to the demographic make-up of Champaign Urbana (approximately 20\%) area with 11 African Americans, 4 Asians, 2 American Indian/Alaskan Native, 4 more than one race in addition to the 97 Caucasian participants. Figure 1 represents the CONSORT and flow of participants through the trial. Figure 2 represents the recruitment and enrollment rates of participants.

Table 1 Baseline Characteristics of Older Adults in Yoga and Stretching-Strengthening Control Groups*.

\begin{tabular}{lll}
\hline & Yoga $(\mathrm{n}=61)$ & Control $(\mathrm{n}=57)$ \\
\hline Age (mean SD) & $62.1( \pm 5.82)$ & $62.0( \pm 5.39)$ \\
$\begin{array}{l}\text { Sex }(\mathrm{n}, \%) \\
\text { Females }\end{array}$ & $49(80.3)$ & $43(75.4)$ \\
Males & $12(19.7)$ & $14(24.6)$ \\
Race (n, \%) & & \\
African American & $7(11.5)$ & $4(7.0)$ \\
Asian & $2(3.3)$ & $2(3.5)$ \\
American Indian/Alaskan Native & $0(0)$ & $2(3.5)$ \\
More than one race & $1(1.6)$ & $3(5.3)$ \\
Caucasian & $51(83.6)$ & $46(80.7)$ \\
& & \\
Education (n, \%) & $14(23)$ & $25(43.8)$ \\
$<$ College degree & $12(19.7)$ & $32(56.2)$ \\
$>$ College degree & & \\
\hline
\end{tabular}

* No significant group differences at baseline. 


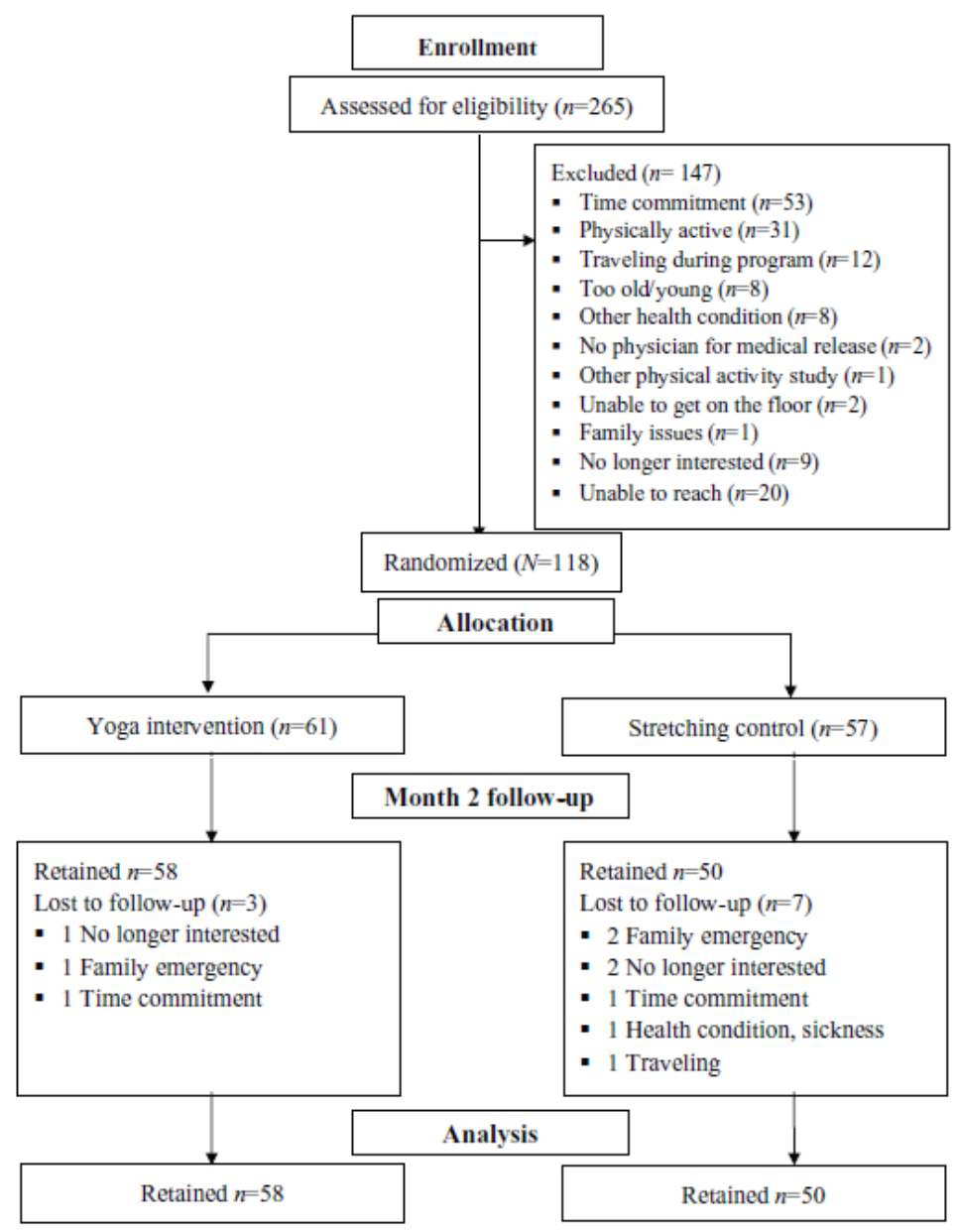

Figure 1 Flow of participants through the study. Reproduced with permission from Gothe et al. in Journals of Gerontology: Medical Sciences [14].

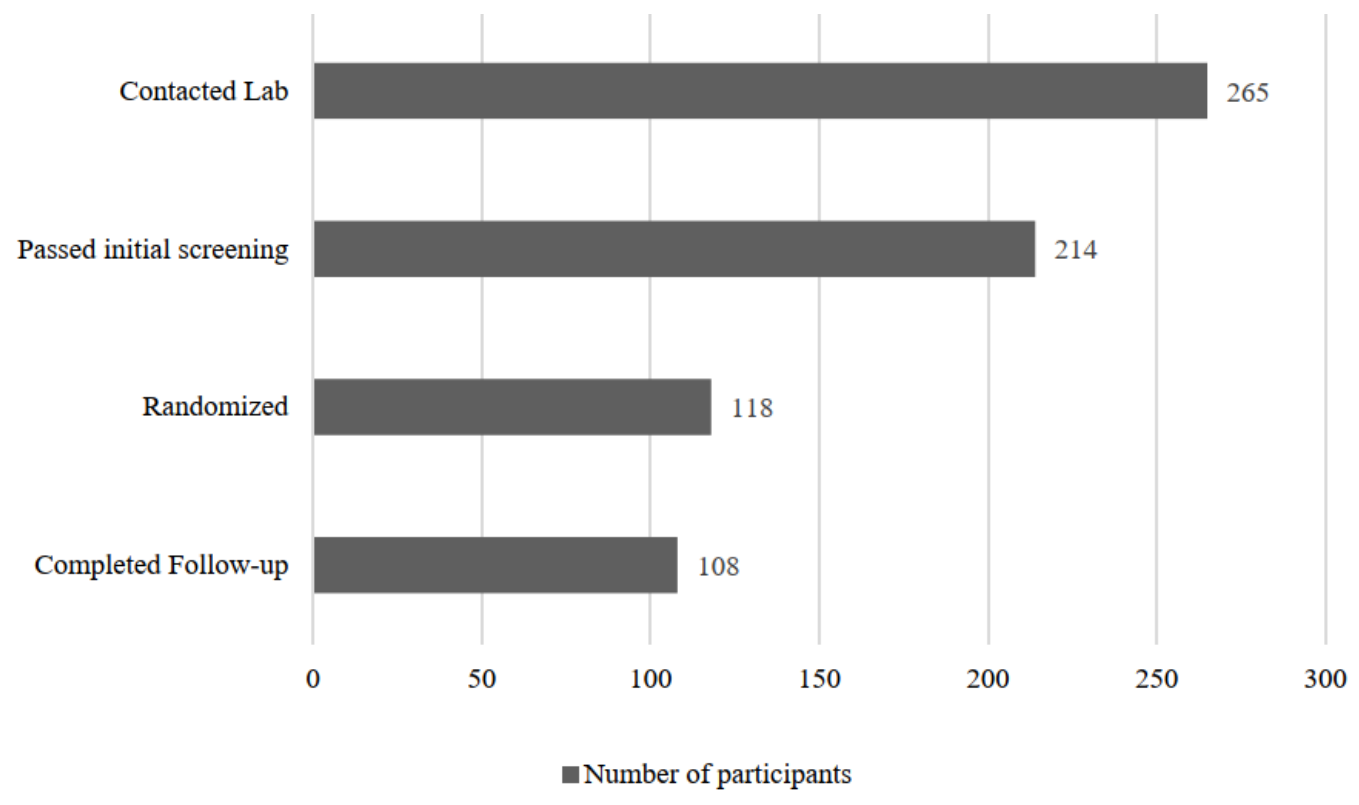

Figure 2 Recruitment and enrollment flow. 


\subsection{Average Intensity of the Exercise Sessions}

Average intensity of the stretching-strengthening group and pose exercises performed in the yoga group is displayed in Figure 3. Both groups exhibited similar changes in the pattern of intensity progression. A Group x Time comparison revealed no significant group differences at any time point ( $p$ values $\geq 0.324$ ).

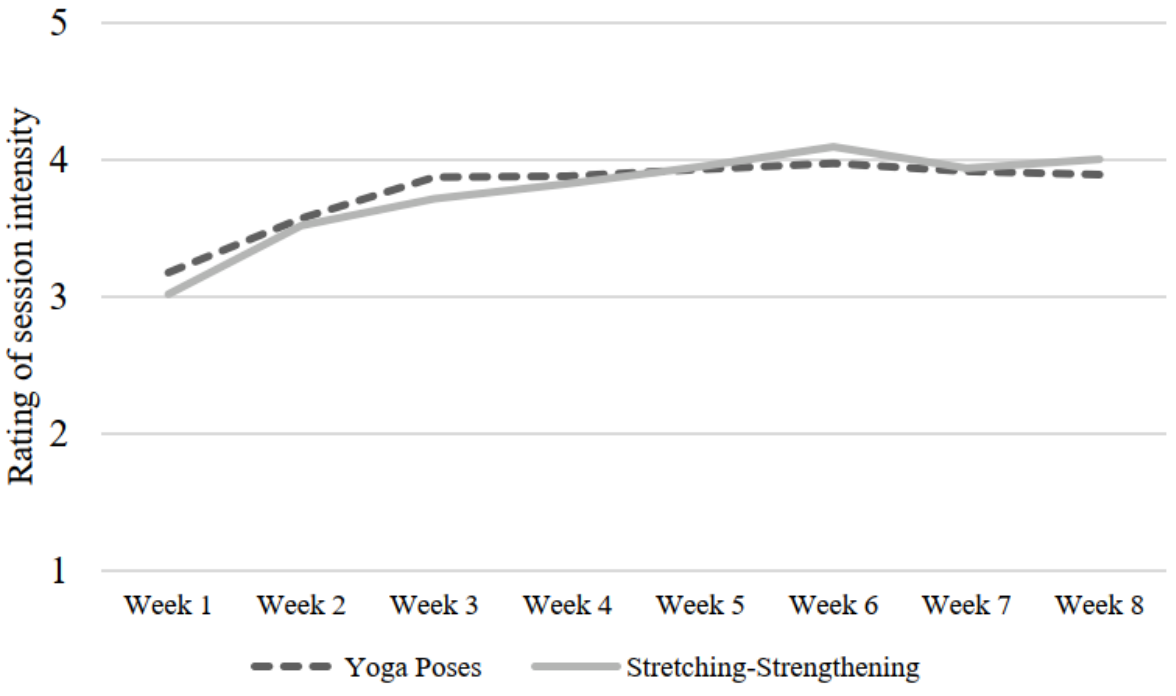

Figure 3 Average ratings for session intensity (based on a 1-5 Likert scale, with 1 representing "very light" and 5 representing "very hard").

\subsection{Intervention Data}

\subsubsection{Average Enjoyment of the Exercise Sessions}

Average session enjoyment for stretching-strengthening and yoga postures is displayed in Figure 4. Enjoyment levels across both groups remained steady over the 8-week duration. A Group x Time comparison revealed no significant group differences ( $p$ values $\geq 0.179$ for each week).

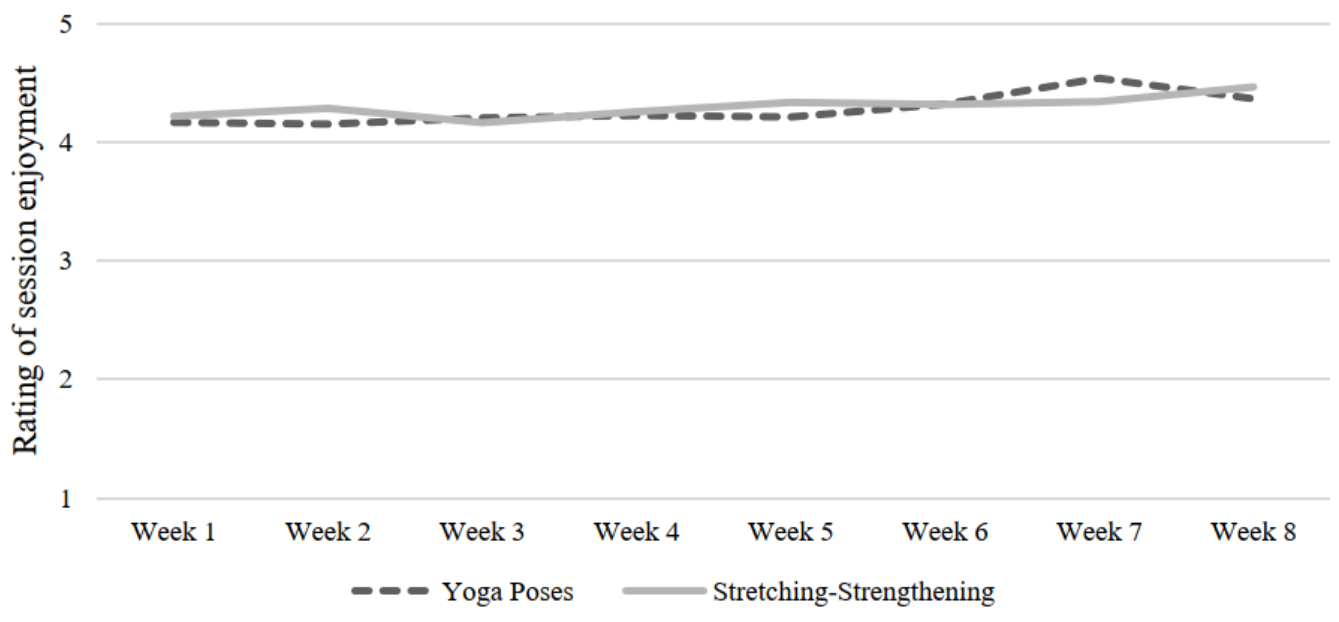

Figure 4 Average ratings for session enjoyment (based on a 1-5 Likert scale, with 1 representing "did not enjoy at all" and 5 representing "very much enjoyed"). 


\subsubsection{Average Perceived Performance during the Exercise Sessions}

Average perceived performance during the stretching-strengthening and yoga postures is displayed in Figure 5. While both groups showed similar trends of improved perceived performance across the 8-week period, there were significant group differences at each time point ( $p$ values $\leqslant$ 0.003). The stretching group universally reported higher average ratings of perceived performance. However, the rate of change in perceived performance across the 8-weeks was greater in the yoga group. The difference between Week 8 and Week 1 scores was 0.86 for yoga and 0.52 for the stretching group.

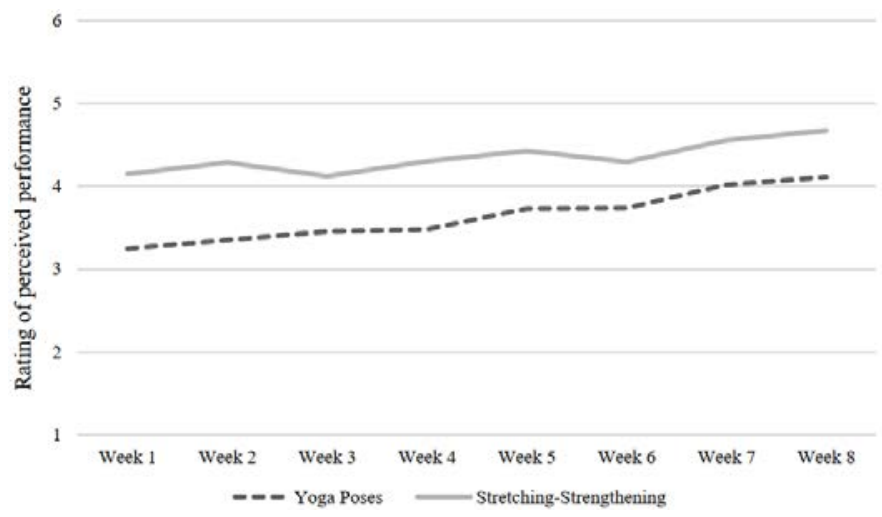

Figure 5 Average ratings for perceived performance per session (based on 1-6 Likert scale, with 1 representing "very poor" and 6 representing "very well").

\subsubsection{Yoga-Specific Breathing and Meditation Ratings}

Each yoga class comprised of 10 minutes each of yoga based breathing exercises (pranayama) and meditative exercises. At the end of each class, participants rated the intensity, enjoyment and perceived performance for these two components across the eight weeks, which is displayed in Figures 6 and 7. Enjoyment ratings for breathing and mediation were steady, showing little variance over the 8-week period. Intensity and perceived performance ratings for breathing and meditation both increased over the 8-weeks.

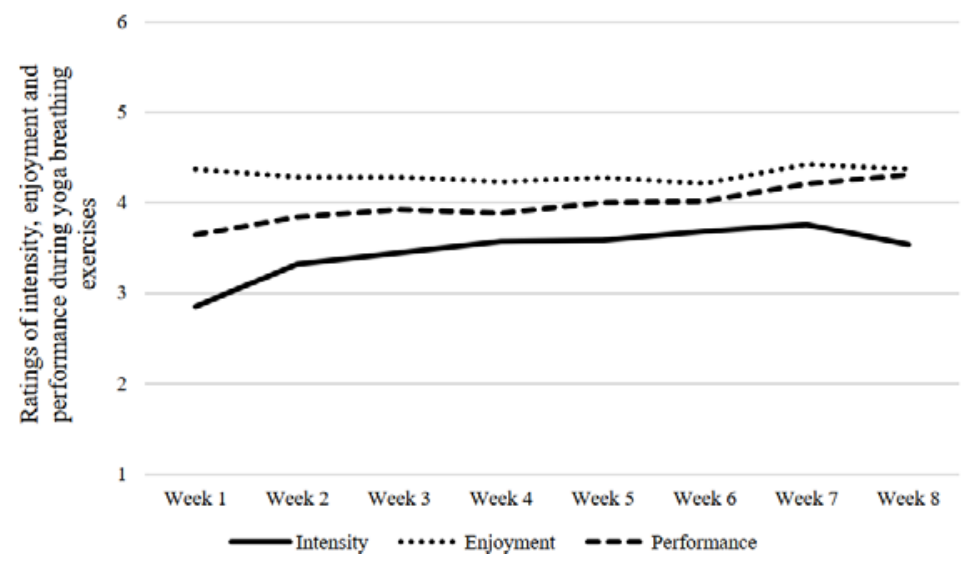

Figure 6 Average ratings of intensity, enjoyment and perceived performance during yoga breathing exercises (based on 1-5 Likert scale for intensity and enjoyment, 1-6 for perceived performance). 


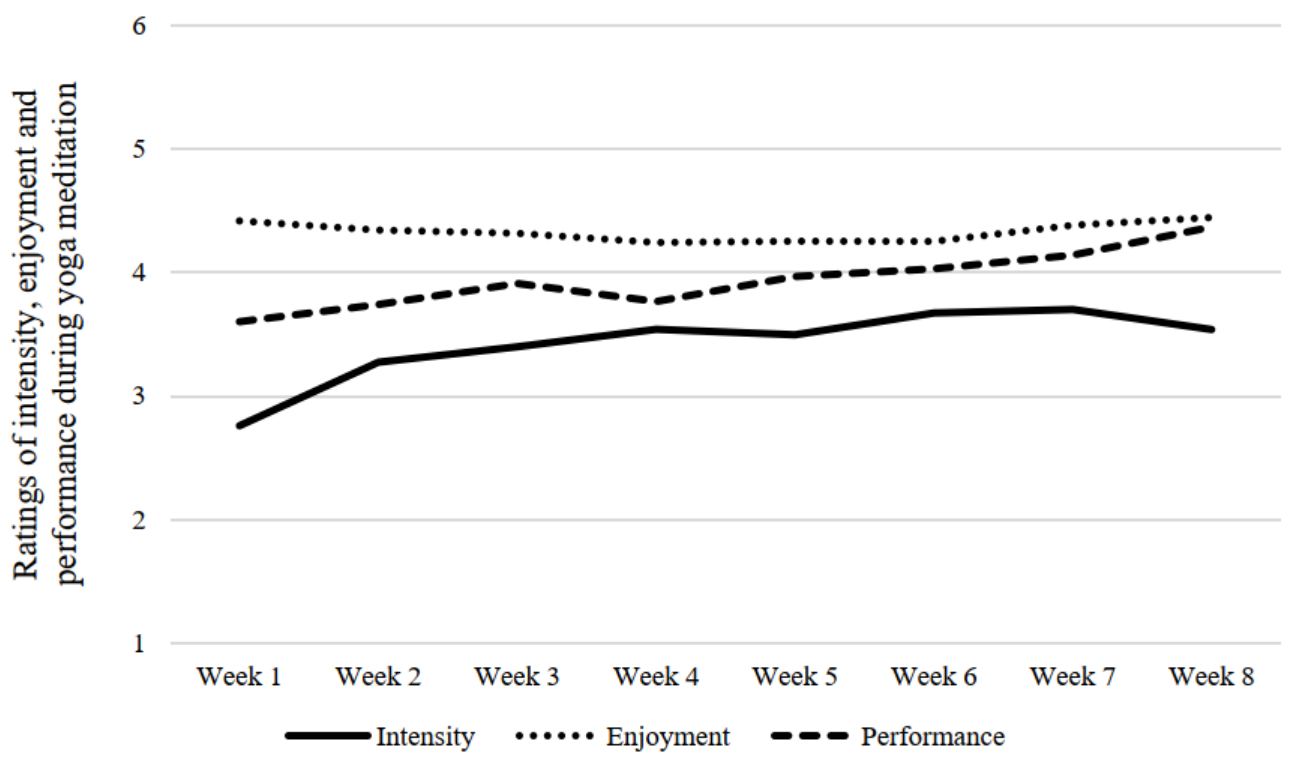

Figure 7 Average ratings of intensity, enjoyment and perceived performance during yoga meditation exercises (based on 1-5 Likert scale for intensity and enjoyment, 1-6 for perceived performance).

\subsection{Attendance, Attrition Rates and Safety}

Both groups met three times per week for 8-weeks, culminating in 24 exercise sessions. The average attendance for the yoga classes was $80.82 \%$ (19.2 \pm 3.8 sessions) and was not statistically different from the average attendance of the stretching classes, which was $81.29 \%(19.4 \pm 3.8$ sessions).

Of the 118 participants who were randomized, 10 dropped out of the study and did not complete follow up assessments. Thus, the total study attrition rate was $8.47 \%$. However, group-specific attrition rates differed. The attrition rate within the yoga group was $4.92 \%$ ( 3 drop-outs) and within the stretching-strengthening group was $12.28 \%$ (7 dropouts). Across all participants who dropped out, "no longer interested" and "family emergency" were the two most cited reasons for discontinued study participation ( $n=3$ for each). "Time commitment" was reported by two participants, and "health condition" and "traveling" were each reported by one participant each.

No adverse events occurred in either group over the entire study period.

\subsection{Participant Program Evaluation}

Out of the 58 yoga participants and 50 stretching-strengthening participants who completed the study, $n=43$ yoga and $n=45$ stretching-strengthening participants completed the participant program evaluation.

On a 1-5 Likert scale, with 5 as the most favorable ("very satisfied") rating, yoga participants gave "exercise program overall" an average rating of $4.86( \pm 0.41)$, "exercises performed" an average rating of $4.74( \pm 0.49)$, "progression of exercises" an average rating of $4.65(0.57)$, and "modifications for exercises" an average rating of $4.62( \pm 0.65)$. Likewise, participants in the stretchingstrengthening group rated their overall experience with the exercise program an average of 4.8 
$( \pm 0.40)$, exercises performed an average rating of $4.58( \pm 0.54)$, progression of exercises an average rating $4.71( \pm 0.51)$, and modifications an average rating of $4.73( \pm 0.54)$.

Regarding program design, participants in both groups cited frequency of classes, instruction quality, and intensity progression as the most beneficial aspects of their 8-week experience. Least beneficial aspects reported among the yoga group included room space and breathing exercises. Among stretching-strengthening participants, the time of the day and the walking warm-up were cited as least beneficial.

When asked to provide suggestions for future exercise classes, participants in both groups suggested a longer program duration (i.e. longer than 8 weeks) and more guided materials (such as exercise DVDs or printed handouts) to continue the exercises after the conclusion of the program.

\section{Discussion}

Based on the participants' average enjoyment, intensity and perceived performance ratings to the exercise sessions, high attendance rates, low study attrition rate and high program satisfaction, our exercise program design is highly feasible and acceptable among older adults.

\subsection{Gender and Racial Differences}

Among the 118 adults enrolled in the study, $77.97 \%$ ( $n=96)$ were females and $82.20 \%(n=97)$ were Caucasian. There were no significant differences between the demographic characteristics of participants who completed the study and those who dropped out. These overproportions are consistent with previous national surveys of yoga practitioners [18-20], who have reported the predominance of Caucasian, female, young and well-educated characteristics among those who regularly practice yoga. Additionally, clinical trials examining the efficacy of yoga to improve psychological conditions (i.e. depression, stress) and quality of life have often reported higher female enrollment [21-23]. Future studies should use targeted advertising and recruitment strategies to appeal to male and minority populations to engage in yoga based interventions.

\subsection{Time Commitment and Adherence to Program}

There are currently no official recommendations for the dosage of yoga needed to produce improvements in physical or mental health; however, the previously published outcomes of the current exercise trial revealed that a dose of 3 hours/week for 8-weeks was enough to significantly improve cognitive function [14, 15] and functional fitness [7]. Nevertheless, this time commitment showed to be a significant deterrent for potential participants, as 53 volunteers declined to participate due to this frequency and dose of the planned intervention. For participants who enrolled in the study, time commitment did not emerge as a burden, as only 2 out of the 10 dropouts cited this as their reason for discontinuing. Our attendance and attrition rates were comparable to previous yoga RCTs with healthy older adults. Flegal et al. [24] conducted a 6-month trial with one 90-minute lyengar yoga session per week and reported a 77\% class attendance rate and $13 \%$ dropout rates. Tiedemann et al. [6] also conducted an lyengar yoga session with healthy older adults, holding two one-hour sessions per week for 12 weeks. Here the authors reported an 83\% attendance rate and $0 \%$ attrition. Iyengar yoga places greater focus on poses, with strong emphasis on precision and alignment of the body [25]; thus, it is possible that the types of yoga (lyengar versus 
Hatha which includes breathing and meditative practice) may influence participant adherence to the intervention.

Additionally, while the exercise sessions for our trial were at predetermined days and times, future studies may benefit from offering participants alternate times (such as a morning or evening session), giving them more flexibility to work the yoga classes into their personal schedules. At the same time, many participants who completed the study expressed interest in continuing the exercise sessions past the 8-week study period. Suggestions from the participants included both increasing the duration of the program (i.e. longer than 8 weeks) and receiving instructional materials to continue the exercises on their own. Thus, there appears to be a subjective preference that could be affected by age, employment status as well as personal factors such as motivation to commit to regular exercise.

\subsection{Perceived Performance}

Across the entire 8-week program, participants in the stretching-strengthening group reported consistently higher ratings for their perceived performance of the exercises. This is not surprising, given that these classes were composed of familiar exercises incorporating simple motions (i.e. bicep curls, squats, standing ab crunches). On the contrary, participants in the yoga group were exposed to more novel exercise poses (i.e. upward salute, warrior, tree pose) requiring the need to learn new movements, which often combined balance and flexibility making it more challenging. The need for flexibility has previously been reported as a perceived barrier to starting yoga for individuals with low flexibility [26]. However, our study showed that the rate of improved perceived performance was greater among the yoga group. This revealed that our program was effective in increasing the abilities of primarily novice older adults to perform yoga poses, which in turn improved their own perception of their performance. Such "mastery experiences" are important components of self-efficacy [27], which is a strong predictor of exercise behavior maintenance [28]. Future yoga interventions should be designed to both acknowledge the hesitance and/or intimidation novice participants' may feel when starting yoga practice, but also capitalize on the opportunity to help participants reach new milestones and accomplishments throughout their practice.

\subsection{Safety Considerations}

While yoga can be modified and adapted for populations with limited mobility (i.e. chair yoga), true Hatha yoga requires a flow of movements requiring participants to frequently move on and off the floor. To allow for the design of our yoga classes to be homogeneous so that all participants primarily engaged in the same poses without the need for extensive modifications, we included a study criterion requiring participants to be 'able to get up and down from the floor'. Although our study recruited older adults, who often face functional limitations preventing frequent postural changes, this study criterion did not negatively affect our recruitment ability. Out of the 265 individuals who were interested in the study, only two were ineligible based on this criterion. Future studies could use this criterion to develop homogenous yoga curricula as many yoga poses, including the corpse pose (shavasana) can only truly be performed on the floor.

No adverse events were reported throughout the study period, which is indicative that our yoga program was safe and well tolerated by our target population. While it has been previously 
suggested that adults $\geq 65$ years of age may be at greater risk for yoga-related injuries [13], many of these risks can be mitigated by thorough health screening, proper instruction by a certified yoga instructor and suitable duration and frequency as demonstrated by our trial.

\subsection{Active Control Group}

Given that the primary aim of our study was to test the efficacy of yoga to improve cognition among older adults, an appropriate control arm was necessary to match the primary elements of a group exercise class. Figures 2 and 3 show that there was no significant difference in exercise intensity or session enjoyment between the yoga and control group. It has been previously suggested that control arms in physical activity RCTs should be designed to allow comparison of the efficacy of one exercise mode over the other to produce a desired outcome [29]. Not only is this approach considered more ethical (as opposed to mandating inactivity), but it falls in line with traditional clinical research of providing "treatment as usual" for the control arms. Our stretchingstrengthening group effectively met this criterion, as participants in this group experienced the same level of social interaction, instructor guidance, session enjoyment and exercise intensity as the participants in the yoga group. Thus, future studies can effectively use stretching-strengthening exercises as an active control arm for yoga based RCTs as the stretching-strengthening exercises mimic the yoga postures.

\subsection{Implications for Future Studies}

Although we aimed to recruit a representative sample of the Champaign-Urbana community, our study participants ended up being predominantly Caucasian and female. However, as previously mentioned, national surveys and previous yoga interventions have shown these to be the primary characteristics of individuals interested in yoga [30]. It is likely that these prevalent characteristics are a byproduct of yoga's commercialization in mainstream Western media [31]. Thus, in order to gain a broader representation of individuals in Western-based yoga research, future research efforts should attempt to shift how yoga is perceived and promoted through the popular media and Western culture. It is also possible that the participant makeup was a factor of our study's location, which was based in a college town. American college towns, defined as communities where a university's presence has a strong influence on the social and industrial cultures, are traditionally composed of residents with higher socioeconomic status than the national averages [32]. We recommend future yoga research build on these lab-based RCTs to develop community-based yoga programs for older adults. Not only would this increase yoga's accessibility to individuals across geographic landscapes but would be a cost-effective approach to promote physical activity among healthy older adults. Developing effective ways to promote physical activity in this population is imperative, given that this is the fastest growing segment of the US population [33]. As our results demonstrate, 8-weeks of Hatha yoga practice was well-tolerated and acceptable for older adults. While we reported feasibility metrics of adherence, attrition, recruitment rates and overall value of the program (as measured by participant ratings) which may be useful for those trying to implement a community-based yoga program, a metric which was not included in our report was cost. In order to effectively scale up yoga programs to make them available on a community level for healthy older 
adults, cost-effectiveness studies are needed to examine the financial implications of a yoga program encompassing: advertising, staffing, equipment, and location needs.

\section{Conclusions}

In conclusion, the feasibility and acceptability data from our RCT are promising and demonstrate that yoga interventions can be safely and successfully executed with novice middle aged and older adults. A stretching-strengthening active control group successfully mimics the yoga postures and can be used by yoga researchers to overcome the drawbacks of having a waitlist or usual care control groups that are more common in the literature. We hope that the feasibility and acceptability data from our preliminary study will aid researchers in designing future yoga interventions that can be successfully implemented and provide robust and clinically useful evidence regarding efficacy and safety of yoga for older adults.

\section{Acknowledgments}

The authors would like to thank yoga instructors Jennifer Allen and Steven Willette, and exercise specialists Jordan Orr and Merril Idiyalil for supervising the exercise groups. A sincere thank you to all the study participants who volunteered their time to participate in this 8-week intervention. Thank you to Drs. Edward McAuley and Arthur Kramer for their mentorship on this project.

\section{Author Contributions}

NG: Conceptualized, designed and conducted the study, conceptualized and wrote the manuscript. EE: Assisted with data analysis and writing of this manuscript.

\section{Funding}

This work was supported by the National Institute on Aging at the National Institutes of Health (2R01 AG20118).

\section{Competing Interests}

The authors have declared that no competing interests exist.

\section{References}

1. Clarke TC, Barnes PM, Black LI, Stussman BJ, Nahin RL. Use of yoga, meditation, and chiropractors among U.S adults aged 18 and over. Hyattsville, MD: National Center for Health Statistics; 2018.

2. Cherniack EP, Senzel RS, Pan CX. Correlates of use of alternative medicine by the elderly in an urban population. J Altern Complement Med. 2001; 7: 277-280.

3. Flaherty JH, Takahashi R, Teoh J, Kim JI, Habib S, Ito M, et al. Use of alternative therapies in older outpatients in the United States and Japan: Prevalence, reporting patterns, and perceived effectiveness. J Gerontol A Biol Sci Med Sci. 2001; 56: M650-M655. 
4. Eisenberg DM, Davis RB, Ettner SL, Appel S, Wilkey S, Van Rompay M, et al. Trends in alternative medicine use in the United States, 1990-1997: Results of a follow-up national survey. JAMA. 1998; 280: 1569-1575.

5. Astin JA, Pelletier KR, Marie A, Haskell WL. Complementary and alternative medicine use among elderly persons: One-year analysis of a Blue Shield Medicare supplement. J Gerontol A Biol Sci Med Sci. 2000; 55: M4-M9.

6. Tiedemann A, O'Rourke S, Sesto R, Sherrington C. A 12-week lyengar yoga program improved balance and mobility in older community-dwelling people: A pilot randomized controlled trial. J Gerontol A Biol Sci Med Sci. 2013; 68: 1068-1075.

7. Gothe NP, McAuley E. Yoga is as good as stretching-strengthening exercises in improving functional fitness outcomes: Results from a randomized controlled trial. J Gerontol A Biol Sci Med Sci. 2016; 71: 406-411.

8. Schmid AA, Van Puymbroeck M, Koceja DM. Effect of a 12-week yoga intervention on fear of falling and balance in older adults: A pilot study. Arch Phys Med Rehabil. 2010; 91: 576-583.

9. Brown KD, Koziol JA, Lotz M. A yoga-based exercise program to reduce the risk of falls in seniors: A pilot and feasibility study. J Altern Complement Med. 2008; 14: 454-457.

10. Chen KM, Tseng WS, Ting LF, Huang GF. Development and evaluation of a yoga exercise programme for older adults. J Adv Nurs. 2007; 57: 432-441.

11. Wang DS. Feasibility of a yoga intervention for enhancing the mental well-being and physical functioning of older adults living in the community. Act Adapt Aging. 2010; 34: 85-97.

12. NCClH. Pilot studies: Common uses and misuses [Internet]. Bethesda, MD: National Center for Complementary and Integrative Health; 2017. Available from: https://www.nccih.nih.gov/grants/pilot-studies-common-uses-and-misuses.

13. Swain TA, McGwin G. Yoga-related injuries in the United States from 2001 to 2014. Orthop J Sport Med. 2016; 4: 2325967116671703.

14. Gothe NP, Kramer AF, McAuley E. The effects of an 8-week Hatha yoga intervention on executive function in older adults. J Gerontol A Biol Sci Med Sci. 2014; 69: 1109-1116.

15. Gothe NP, Kramer AF, McAuley E. Hatha yoga practice improves attention and processing speed in older adults: Results from an 8-week randomized control trial. J Altern Complement Med. 2017; 23. 35-40.

16. Eldridge SM, Lancaster GA, Campbell MJ, Thabane L, Hopewell S, Coleman CL, et al. Defining feasibility and pilot studies in preparation for randomised controlled trials: Development of a conceptual framework. PLoS One. 2016; 11: e0150205.

17. U.S Census Bureau. American Community Survey, 2012 American Community Survey 1-Year Estimates, Table DP05 [Internet]. 2012 [cited date (2020 Oct 8)]. Available from: https://data.census.gov/cedsci/table?q=United\%20States\&tid=ACSDP1Y2012.DP05.

18. Ross A, Friedmann E, Bevans M, Thomas S. National survey of yoga practitioners: Mental and physical health benefits. Complement Ther Med. 2013; 21: 313-323.

19. Birdee GS, Legedza AT, Saper RB, Bertisch SM, Eisenberg DM, Phillips RS. Characteristics of yoga users: Results of a national survey. J Gen Intern Med. 2008; 23: 1653-1658.

20. Cramer H, Ward L, Steel A, Lauche R, Dobos G, Zhang Y. Prevalence, patterns, and predictors of yoga use: Results of a U.S. nationally representative survey. Am J Prev Med. 2016; 50: 230-235.

21. Chong CS, Tsunaka M, Tsang HW, Chan EP, Cheung WM. Effects of yoga on stress management in healthy adults: A systematic review. Altern Ther Health Med. 2011; 17: 32-38. 
22. Cramer H, Anheyer D, Lauche R, Dobos G. A systematic review of yoga for major depressive disorder. J Affect Disord. 2017; 213: 70-77.

23. Patel NK, Newstead AH, Ferrer RL. The effects of yoga on physical functioning and health related quality of life in older adults: A systematic review and meta-analysis. J Altern Complement Med. 2012; 18: 902-917.

24. Flegal KE, Kishiyama S, Zajdel D, Haas M, Oken BS. Adherence to yoga and exercise interventions in a 6-month clinical trial. BMC Complement Altern Med. 2007; 7: 37.

25. Iyengar BKS. BKS Iyengar yoga: The path to holistic health. London: Penguin; 2007.

26. Atkinson NL, Permuth-levine R. Benefits, barriers, and cues to action of yoga practive: A focus group approach. Am J Health Behav. 2009; 33: 3-14.

27. Bandura A. Self-efficacy. Encyclopedia of human behavior. Cambridge, MA: Academic Press; 1994. p.71-p.81.

28. McAuley E. Self-efficacy and the maintenance of exercise participation in older adults. J Behav Med. 1993; 16: 103-113.

29. Huffman KM, Slentz CA, Kraus WE. Control arms in exercise training studies: Transitioning from an era of intervention efficacy to one of comparative clinical effectiveness research. J Appl Physiol. 2011; 111: 946-948.

30. Park CL, Braun T, Siegel T. Who practices yoga? A systematic review of demographic, healthrelated, and psychosocial factors associated with yoga practice. J Behav Med. 2015; 38: 460471.

31. Bhalla N, Moscowitz L. Yoga for every (body)? A critical analysis of the evolution of yoga representation across four decades in yoga journal. J Mag Media. 2019; 19: 75-103.

32. Gumprecht B. The American college town. Geogr Rev. 2003; 93: 51-80.

33. CDC. Promoting health for older adults [Internet]. Atlanta, GA: National Center for Chronic Disease Prevention and Health Promotion; 2020. Available from: https://www.cdc.gov/chronicdisease/resources/publications/factsheets/promoting-healthfor-older-adults.htm.

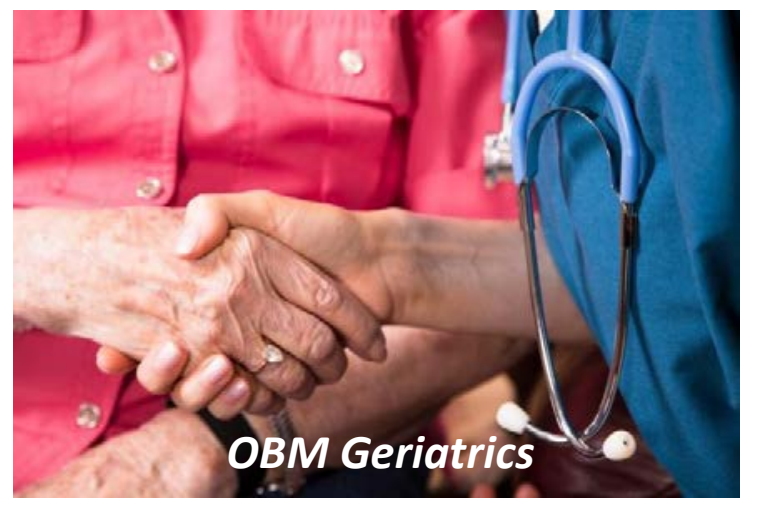

Enjoy OBM Geriatrics by:

1. Submitting a manuscript

2. Joining in volunteer reviewer bank

3. Joining Editorial Board

4. Guest editing a special issue

For more details, please visit: http://www.lidsen.com/journals/geriatrics 